\title{
Numerical Investigation on Gas-solid Flow in a Circumfluent Cyclone Separator
}

\author{
Pan Zhang ${ }^{1}$, Jihai Duan ${ }^{2}$, Guanghui Chen ${ }^{2}$, Weiwen Wang ${ }^{2 *}$ \\ ${ }^{1}$ College of Electromechanical Engineering, Qingdao University of Science and Technology, Qingdao 266069, China \\ ${ }^{2}$ College of Chemical Engineering, Qingdao University of Science and Technology, Qingdao 266043, China
}

\begin{abstract}
The efficient removal of fine dust from exhaust gas reduces the harm to human health and minimizes material waste. A circumfluent cyclone (CFC) was designed to perform with increased gas-solid separation efficiency and decreased pressure drop compared to conventional cyclone separators, and its flow pattern and velocity distribution were investigated. Commercial computational fluid dynamics software was used to simulate the 3D-gas-flow field using the Reynolds stress model (RSM). The simulation results (e.g., for the velocity profile, pressure drop, and separation efficiency) were in good agreement with the experimental results and indicated that about $78 \%$ of the gas flows directly out of the CFC through the inner cylinder and then the vortex finder, which significantly shortens the cleaned gas flow path and thereby reduces friction loss. The CFC also decreases the shortcut flow rate near the vortex finder entrance, reducing particle escape. Finally, the discrete particle model (DPM) was used to predict the flow pattern of particles of different sizes.
\end{abstract}

Keywords: Circumfluent cyclone; Flow pattern; Separation efficiency; Pressure drop.

\section{INTRODUCTION}

Cyclone separators, which separate dispersed solid particles from a fluid suspension by centrifugal and vortex action, have played an important role in industry for the separation and recovery of fine particles. For a cyclone separator, collection efficiency and pressure losses are the main performance characteristics.

Numerous studies have shown that many factors can affect the separation performance of separators, such as the geometric structure and dimension of a cyclone separator (Liu et al., 2018), particles size and distribution (Ma et al., 2015), particle loading (Lin et al., 2018). Studies have been conducted to evaluate geometric effects on conventional cyclones, typically of the Stairmann and Lapple types (Lapple, 1951; Stairmand, 1951). For example, literature surveys (Avci and Karagoz, 2003; Lim et al., 2004b; Qian and Zhang, 2005) showed that the vortex length is vitally important for the prediction of collection efficiency. Gimbum et al. (2005) and Chuah et al. (2006) evaluated the effects of cone tip diameter on the collection efficiency and pressure drop of gas cyclones. Lim et al. (2004a) and Raoufi et al. (2008a) studied the effects of the vortex finder shape and diameter on cyclone performance, also reported

\footnotetext{
${ }^{*}$ Corresponding author.

Tel.: $086+532+84022752$

E-mail address: wwwang@qust.edu.cn
}

by El-Batsh (2013); Elsayed and Lacor (2013) and Brar et al. (2015a). The effects of inlet type on cyclone performance characteristics have been widely investigated, including single and double inlets (Lim et al., 2003; Zhao et al., 2004), tangential inlet and spiral inlets (Zhao et al., 2004), inlet dimensions (Yang et al., 2013), different inlet section angles in relation to the cyclone body (Bernardo et al., 2006; Misiulia et al., 2017), and symmetrical inlets with a volute scroll outlet (Martignoni et al., 2007). In addition, more parameters, such as cyclone height (Xiang and Lee, 2005), dust outlet (Obermair et al., 2003), conical length (Yoshida et al., 2012; Brar et al., 2015b; Hamdy et al., 2017), inlet temperature (Siadaty et al., 2017) and double conical section (Yoshida et al., 2001) have been considered in detail. Furthermore, various new cyclone models have been developed to improve performance parameters, such as the Lee type (Kim and Lee, 1990), semi-spherical cyclone (Ogawa et al., 1991), PV type (Tian et al., 1992), PoC type (Plomp et al., 1996), reverse flow cyclone (Jo et al., 2000), dynamic cyclone (Jiao et al., 2008), and square cyclone (Raoufi et al., 2009). Researchers have also proposed some changes in cyclone structure to improve collection efficiency. Abrahamson et al. (2002) installed different upstream bends in the feed pipe. Chmielniak et al. $(2000,2001)$ applied a vortex baffle to reduce the effect of vortices on separation efficiency. These research show that both the dimensions and the shapes of the cyclone separators all affect the separation performance. It means that the separation performance improvement could be achieved by optimizing cyclone structural or dimensions, or by a new structure design. 
Based on the idea, we have designed a novel cyclone separator, called circumfluent cyclone (CFC), which has two differences in configuration compared with a conventional cyclone: (1) a concentric internal cylinder is installed in the main cylinder, and (2) the inlet duct is located at the bottom of the shell in the CFC rather than at the upper section in the conventional one. In our previous work (Wang et al., 2010), we reported the structure and separation performance of CFC. Our experimental results showed that the $\mathrm{CFC}$ had a higher separation efficiency and a lower pressure drop than the common one. It is well known that the velocity distribution and the flow pattern in a cyclone are closely related to its performance (Hsu et al., 2014). In order to understand the separation mechanism, we focus on the flow pattern and the velocity distribution in the CFC in this work.

Numerical simulations have been widely used to predict the flow field characteristics inside a cyclone (Griffiths and Boysan, 1996). They provide better fundamental understanding and process optimization (Boysan et al., 1982; Zhou and Soo, 1990; Chuah et al., 2006; Gupta and Kumar, 2007). The key to the success of numerical simulations lies with the accurate description of the turbulent behaviour of the flow (Griffiths and Boysan, 1996). The standard $k-\varepsilon$, RNG $k-\varepsilon$ and realizable $k-\varepsilon$ models are not optimized for strong-vortex flows found in cyclones (Bhaskar et al., 2007). Reynolds stress model (RSM) and large-eddy simulations (LES) are only effective turbulent models to simulate vortex flow in cyclones (Derksen and Van den Akker, 2000; Slack et al., 2000; Chuah et al., 2006; Zhao et al., 2006; Bhaskar et al., 2007). In general, RSM can yield an accurate prediction of vortex flow pattern, axial velocity, tangential velocity and pressure drop in cyclone simulations (Hoekstra et al., 1999; Chuah et al., 2006;
Zhao et al., 2006; Bhaskar et al., 2007). LES has also been used to simulate vortex flow in cyclones (Derksen and Van den Akker, 2000; Slack et al., 2000). Because large eddies are resolved directly while small eddies are modelled in LES, the coherent fluctuations in cyclones can be explicitly resolved by LES as long as the resolution is sufficiently high. However, LES needs excessive computational effort and much CPU time. Moreover, recent studies also suggest that RSM can improve the accuracy of the numerical solution. Thus, RSM model was chosen in the all numerical tests in this work.

In the present work, commercial computational fluid dynamics (CFD) software (FLUENT 14.0) was used to simulate the flow pattern and the velocity profiles of CFC. The computational investigation on the effect of the angle of the cone was also conducted.

\section{EXPERIMENTAL SETUP}

The main configuration of a CFC is shown in Fig. 1(a) and 1(b). The cyclone's main dimensions are shown in Table 1. There are two configuration differences between a $\mathrm{CFC}$ and a conventional cyclone. One is that the CFC has an inner cylinder, which is situated in the main cylinder and plays a major role in the collection of fine particles. The other one is that the inlet duct is located at the bottom of the main cylinder in the CFC, rather than at the upper part in a conventional cyclone.

The experiment procedure was that the fly ash in the feed hopper was blown into the gas pipe by pressure air, then dispersed and mixed with the gas from the air blower. The weights of the collected dust in the filter bag and dust hopper were then measured, by which the total collection efficiency of the cyclones can be calculated. The total

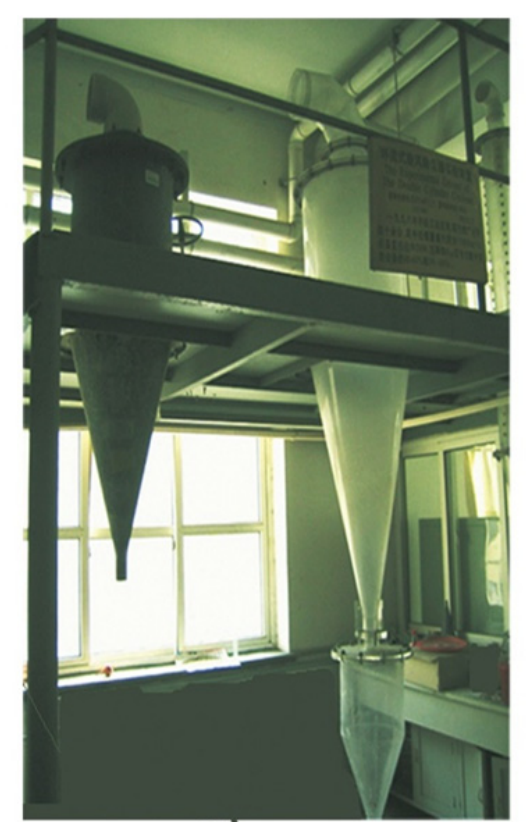

a)

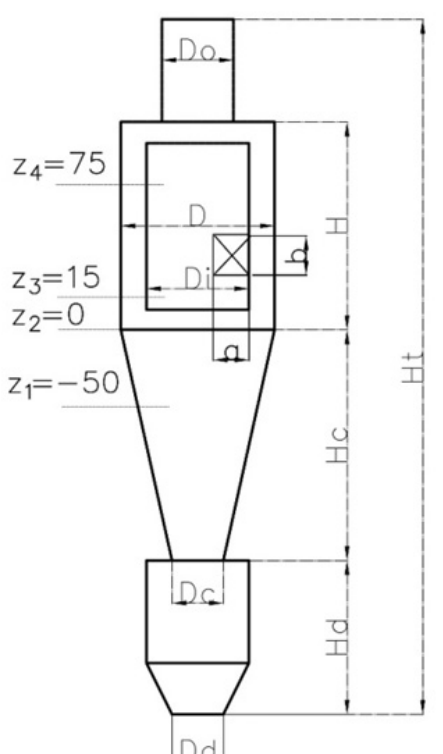

b)

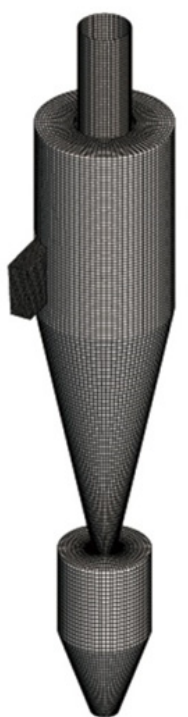

c)

Fig. 1. CFC configuration and grids. (a) Physical map, (b) geometrical dimensions, and (c) cyclone separator mesh. Units in $\mathrm{mm}$. 
Table 1. The main dimensions of the CFC.

\begin{tabular}{lllllllll}
\hline Dimension $(\mathrm{mm})$ & $a$ & $b$ & $D$ & $H$ & $D o$ & $D i$ & $H c$ & $D c$ \\
\hline CFC & 25 & 50 & 138 & 240 & 50 & 90 & 365 & 50 \\
\hline
\end{tabular}

cyclone separation efficiency, $\eta$, is the percentage of the collected particles in dust hopper from the total particles entered into the cyclone. The mean size of fly ash particle diameter was approximately $10.2 \mu \mathrm{m}$; the density of the particles is $2800 \mathrm{~kg} \mathrm{~m}^{-3}$; and the solid loadings of particles are $15 \mathrm{~g} \mathrm{~m}^{-3}$. At the same time the pressure-drops over the cyclones were also measured by using a $\mathrm{U}$ tube water manometer. More detailed testing methods and procedures can be found in our previous work (Wang et al., 2010).

\section{NUMERICAL SETUP}

\section{Turbulence Method}

FLUENT 14.0 was used to carry out calculations with reference to the conservation of mass and momentum equations. The conservation equation for mass in an incompressible Newtonian flow is:

$$
\frac{\partial \rho}{\partial t}+\frac{\partial}{\partial x_{i}}\left(\rho U_{i}\right)=0
$$

where $\rho$ is the fluid density, $U$ is the fluid mean velocity, and the subscripts $i$ denote direction. For the turbulent flow in the cyclones, the RSM was used in this study. The Reynolds stress transport equation is written as:

$$
\begin{aligned}
& \frac{\partial}{\partial t}\left(\rho \overline{u_{i} u_{j}}\right)+\frac{\partial}{\partial x_{k}}\left(\rho U_{k} \overline{u_{i} u_{j}}\right)= \\
& -\frac{\partial}{\partial x_{k}}\left[\rho \overline{u_{i} u_{j} u_{k}}+\overline{p\left(\delta_{k j} u_{i}+\delta_{i k} u_{j}\right)}\right]+\frac{\partial}{\partial x_{k}}\left[\mu \frac{\partial}{\partial x_{k}} \overline{u_{i} u_{j}}\right] \\
& -\rho\left(\overline{u_{i} u_{k}} \frac{\partial U_{j}}{\partial x_{k}}+\overline{u_{j} u_{k}} \frac{\partial U_{i}}{\partial x_{k}}\right)+p \overline{\left(\frac{\partial u_{i}}{\partial x_{j}}+\frac{\partial u_{j}}{\partial x_{i}}\right)}-2 \mu \frac{\partial u_{i}}{\partial x_{k}} \frac{\partial u_{j}}{\partial x_{k}}
\end{aligned}
$$

where $u$ is the fluid fluctuating velocity. The two terms on the left-hand side are the partial time derivative of stress and the convective transport term, respectively. And the first and the second terms on the right-hand side are the stress diffusion terms, while the others are the stress production term, the pressure strain and the dissipation term in sequence.

\section{Particle Equation of Motion}

To calculate the trajectories of particles in the flow, the discrete phase model (DPM) was used to track individual particles through the continuum fluid. The particle loading in the cyclone separator is typically small (less than $12 \%$ volume fraction). Therefore, it can be safely assumed that the presence of the particles does not affect the flow field (one-way coupling) (Chuah et al., 2006; Raoufi et al., 2008b). The equation of motion for an individual particle, including the effects of nonlinear drag and gravitational forces, can be written as:

$$
\frac{d u_{p}}{d t}=F_{D}\left(u-u_{p}\right)+g_{x}\left(\rho_{p}-\rho\right) / \rho_{p}
$$

where $u_{p}$ is the particle velocity, $\rho$ is the fluid density, $\rho_{p}$ is the density of the particle, and $F_{D}$ is the drag force, defined as:

$$
F_{D}=\frac{18 \mu}{\rho_{p} D_{p}^{2}} \frac{C_{D} \operatorname{Re}}{24},
$$

where $\mu$ is the molecular viscosity of the fluid, $D_{p}$ is the particle diameter, and $C_{D}$ is the drag force coefficient for the particles, and the Reynolds number, Re, is:

$$
\operatorname{Re}=\frac{\rho D_{p}\left|u_{p}-u\right|}{\mu} .
$$

In FLUENT, the drag coefficients for spherical particles and non-spherical particles are calculated using the different correlations developed by Alexander and Levenspiel (Fluent Inc., 2005). The virtual mass force, the Basset force, the Saffman force, the Brownian force and the Magnus force can be neglected since the particles has a much larger density than the gas phase. Once the particle velocity field is calculated from the force balance discussed above, the trajectories of all the particles can be obtained using the following equation:

$\frac{d x}{d t}=u_{p}$

The collection efficiency was obtained by releasing a specified number (about 1140) of monodispersed particles at the inlet of the CFC and monitoring the number escaping through the outlet (Jiao et al., 2008). Collisions between the particles and walls of the CFC were assumed to be perfectly elastic.

\section{Numerical Method}

The momentum equations in fluid flows are expressed in terms of non-linear partial differential correlations. The finite volume method was used to discretize the partial differential equations of the model using the SIMPLE (Semi-Implicit Method for Pressure-Linked Equations) method for pressure-velocity coupling. The segregated solution algorithm was selected. The geometry and the mesh were created using the Design Modeler (DM) package of the ANSYS Workbench, starting from its primitives. The 
computational grid was defined by hexahedral cells, nonuniformly distributed, with a total of 676,500 cells. Numerical tests were carried out using 41,300, 676,500 and 822,600 cells to determine the effect of mesh size on the calculated results. The results show that the differences in predicted velocities with 676,500 and 822,600 cells are negligible. The body-fitted technique was used once the grid system was established. The numerical calculation was made with the numerical grids shown in Fig. 1(c). The second-order upwind QUICK scheme was used to discretize the convection term in the model. For pressure, linear discretization was used. A convergence criterion of 1.0E-05 was used for continuity in the $\mathrm{x}, \mathrm{y}$ and $\mathrm{z}$ momentum equations, and the $\mathrm{k}, \varepsilon$ and other stress equations.

\section{Boundary Conditions}

Derksen (2003) established that the simulation accuracy is not affected by the inlet gas flow profile in terms of average velocities and fluctuation levels. Therefore, uniform inlet boundary conditions were assumed in this work. The inlet gas velocity and the particle velocity were both constant values. The boundary conditions at the outlet of the CFC were prescribed by zero stream-wise gradients for all flow variables. For gas phase, no-slip conditions were assumed at the wall. And for solid particles, a reflect condition was used at the wall, and the trap conditions were used at the dustbin wall and at the down flow. The air density is $1.0 \mathrm{~kg} \mathrm{~m}^{-3}$, and the dynamic viscosity is $1.8 \mathrm{E}-5 \mathrm{~Pa} \cdot \mathrm{s}$. The turbulent intensity equals $5 \%$ and the characteristic length is set to be 0.07 times the inlet width (Elsayed and Lacor, 2010).

\section{Verification}

In order to verify the obtained results, we compared the predictions with the measured tangential velocity profiles at some axial stations using Hot-Wire Anemometers (HWA). The present simulation was compared with the measured tangential velocity profiles at some axial sections on the $y-z$ plane (shown in Fig. 1). Fig. 2 shows that RSM simulation basically matches the experimental velocity profile. Considering the complexity of the fluid flow in the cyclones, the agreement between the predictions and measurements is considered quite acceptable.

\section{RESULTS AND DISCUSSION}

\section{Flow Field in CFC}

Fig. 3 illustrates the flow pattern in the CFC. Figs. 3(a), 3(b), 3(c) and 3(d) plot the velocity vectors, pressure distribution, tangential velocity and axial velocity,
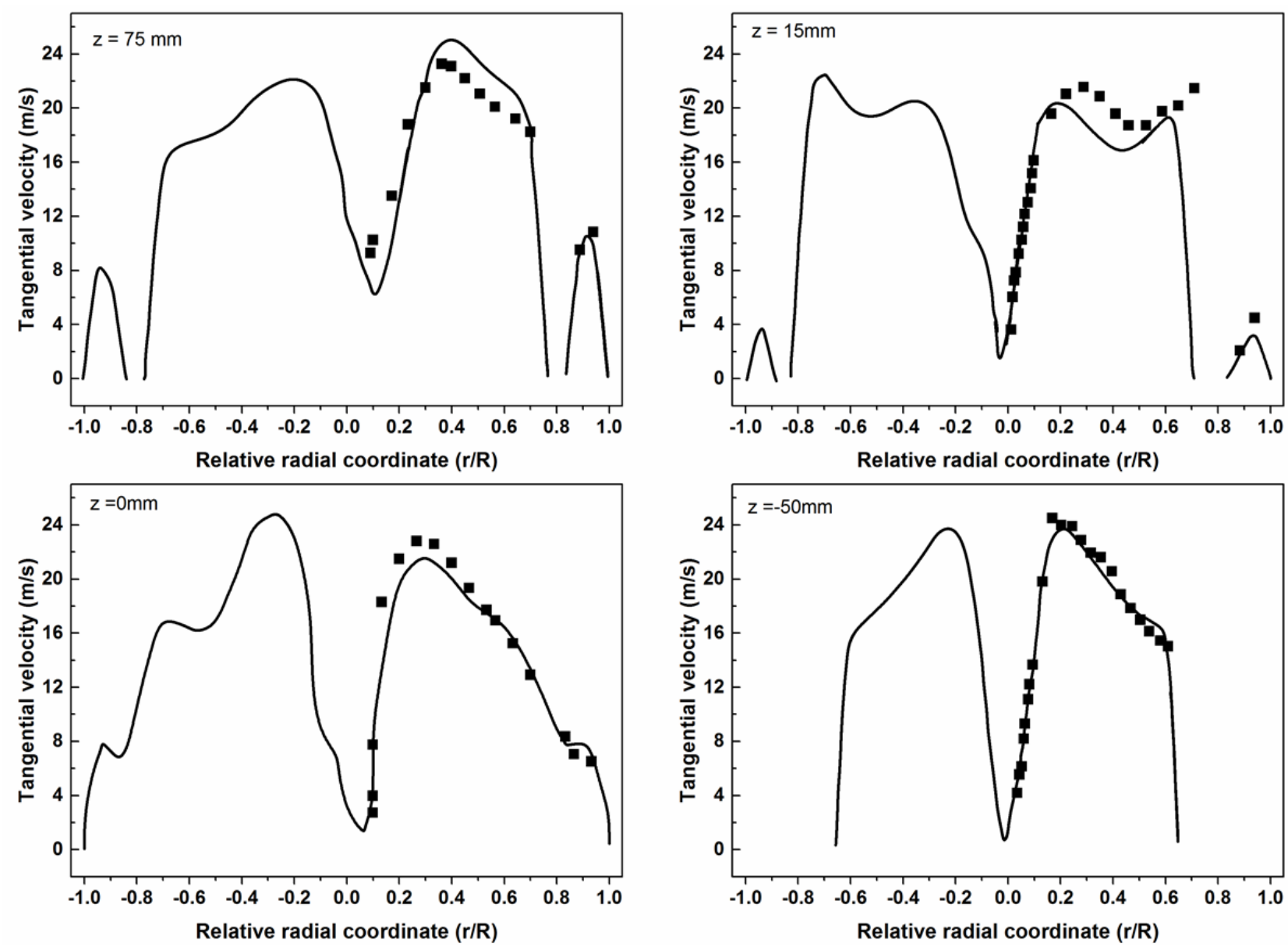

Fig. 2. Comparison of the time-averaged tangential velocity between the HWA measurements and the calculated results at some sections on the $\mathrm{y}-\mathrm{z}$ plane. (—: model; $\mathbf{a}$ : experimental) 




a)
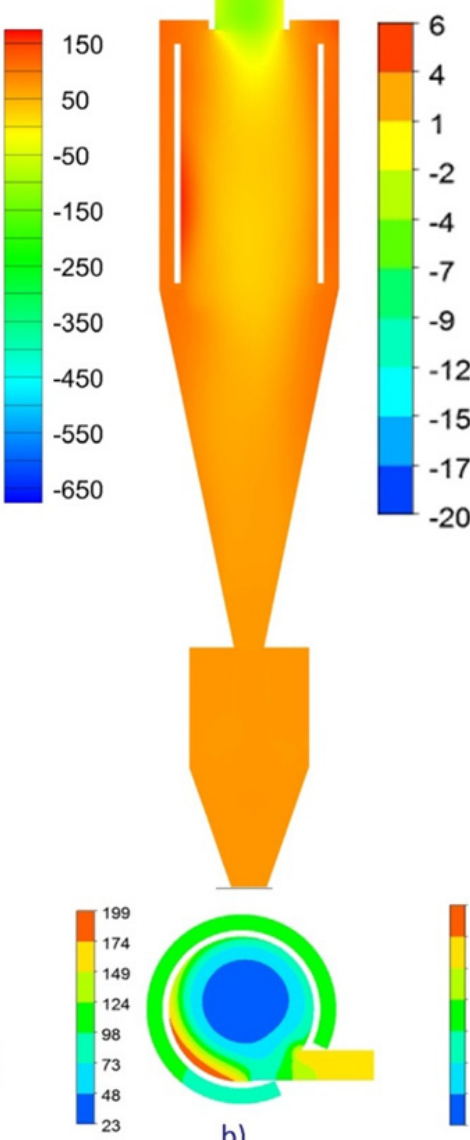

b)

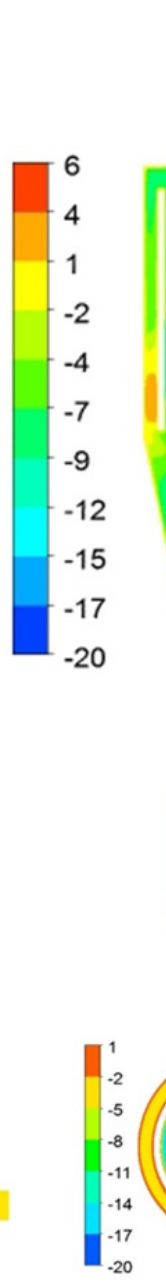

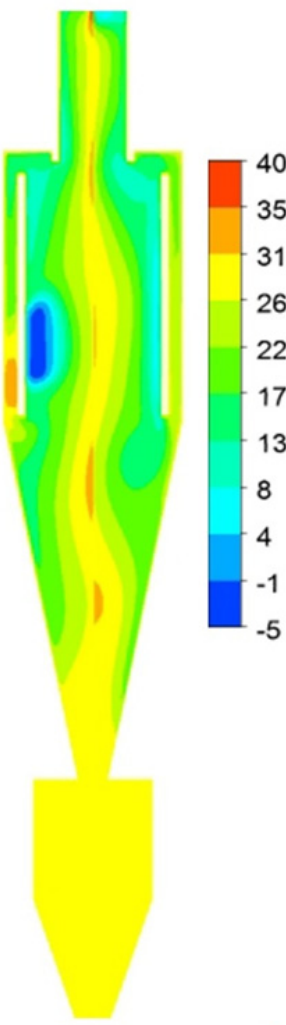

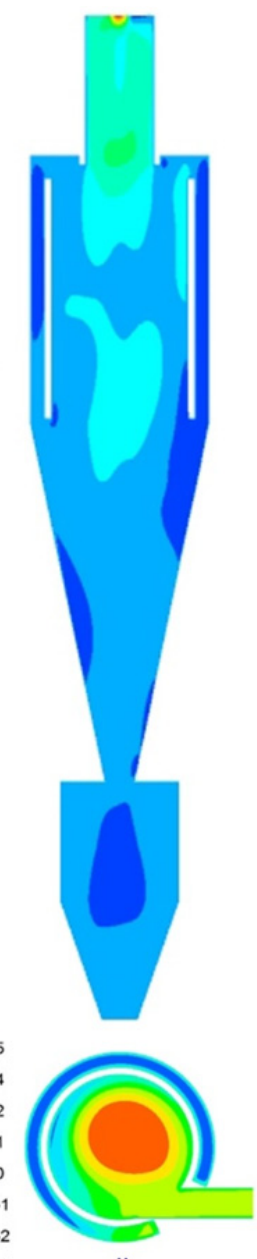

d)

Fig. 3. The velocity vector and the contour plots for the time-averaged flow variables. (a) Velocity vector, (b) pressure distribution, (c) tangential velocity, and (d) axial velocity. Top: the vertical plane $\mathrm{x}=0$, bottom: $\mathrm{z}=20 \mathrm{~mm}$.

respectively, on the vertical plane $\mathrm{x}=0 \mathrm{~mm}$ at the inlet velocity of $19 \mathrm{~m} \mathrm{~s}^{-1}$. As shown in Fig. 3, the gas flows first enter the inner cylinder and the vortex flow generated by the tangential inlet moves upward, meanwhile, most of the particles would be separated from the rotating gas flow. The upward flow divides into two parts when the vortex flow comes near the bottom of the vortex finder. One part, the pure separated gas, directly flows out of the CFC through the vortex finder. The other one with the fly ash particles flows into the annular space between the inner cylinder and the main cylinder, and continues to flow downward to the cone zone, in which the air is further separated from the dust. Thus, the CFC provides a new separation approach, different from that in a common cyclone.

The time-averaged static pressure decreases radially from the wall to the centre. A low-pressure zone appears in the central forced vortex region due to high swirling velocity. The flow field indicates the expected quasiforced/quasi-free combination of the Rankine type vortex, with the inner vortex behaving like solid body rotation and the outer vortex behaving like the rotation of an inviscid fluid (Brar et al., 2015b). The difference is that two vortex motions all move up in the main cylinder, rather than the outer vortex moving down and the inner vortex moving up as in a common cyclone (Wang et al., 2006).

\section{Shortened Gas Flow Path in CFCs}

Figs. 4(a) and 4(b) show the sketch map of the flow pattern in the CFC and in a common cyclone, respectively. As shown in the figure, the gas $\left(Q_{\text {in }}\right)$ flows into the inner cylinder (Zone A in Fig. 4) and comes together with the flow $\left(\mathrm{Q}_{2}\right)$ from the cone, then spirals upwards in the CFC. The upward flow separates into two parts around the bottom of the vortex finder. The major part $\mathrm{Q}_{\text {out }}$ (see Fig. 4(a)), about $78 \%$ [ $\left.=\mathrm{Q}_{\text {out }} /\left(\mathrm{Q}_{\text {out }}+\mathrm{Q}_{2}\right)\right]$, directly flows out of the $\mathrm{CFC}$ through the vortex finder. It means that the flow path of the main part of the gas only traverses the inner cylinder and the vortex finder. It is considerably shorter than the path in a convention cyclone, in which it traverses a complicated route through the main cylinder zone, the cone zone and the vortex finder (see Fig. 4(b)) based on the results in the literature (Derksen, 2003; Lee et al., 2006). This should indicate that the circumfluent cyclone (CFC) can reduce the air path, and thus decrease the friction loss and the pressure drop. 


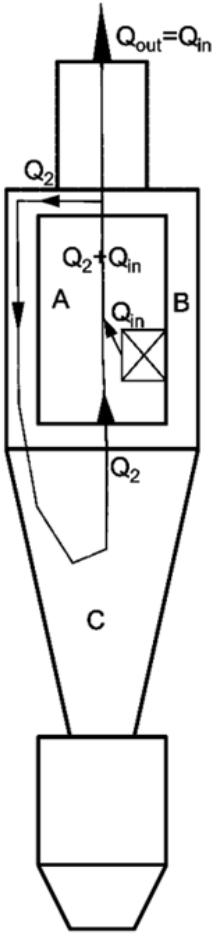

(a)

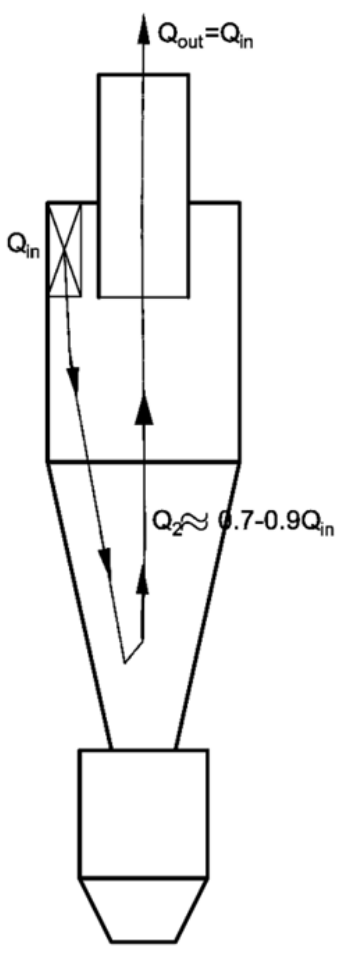

(b)
Fig. 4. The sketch map of the flow pattern. (a) CFC and (b) a common cyclone.

\section{Improved Cut Flow in CFCs}

Fig. 5 shows the velocity vector near the vortex finder entrance in the CFC Zone A and a common cyclone (Xu et al., 2016). Many studies showed that there is a shortcut flow at the entrance of the vortex finder in a reverse-flow cyclone separator (Derksen and Van, 2000; Xiang and Lee, 2005). Moreover, the shortcut flow is significant, up to

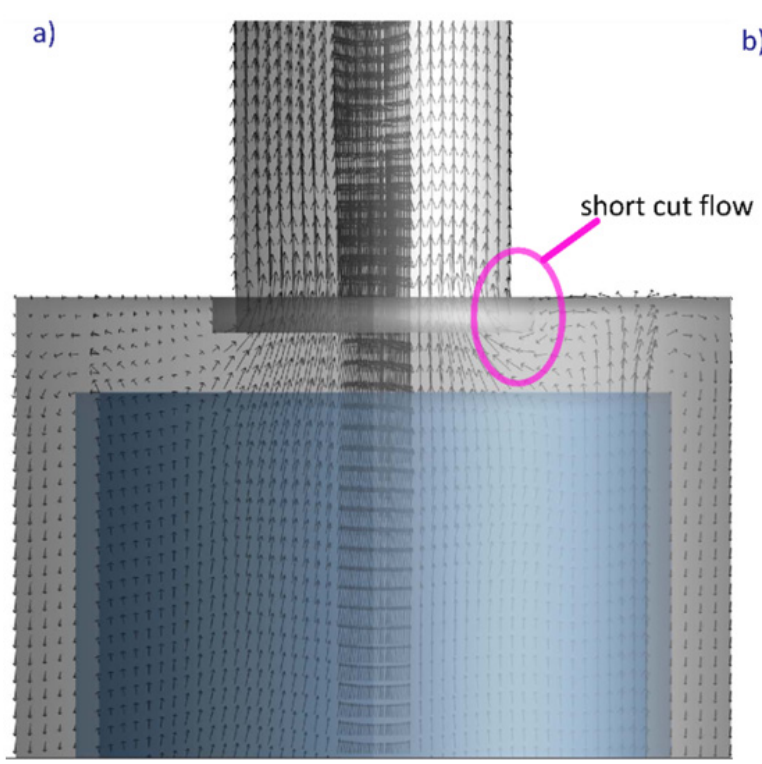

roughly $38 \%$ of the inlet flow rate, which escapes from the separator through the vortex finder inlet in a common cyclone separator (Xu et al., 2016). Fig. 5(a) shows the gas flow in the CFC vortex finder. Most clean gas enters the vortex finder, while the gas containing particles moves to Zone B (see Fig. 4(a)). There is little shortcut flow in the CFC separator. This indicates that the CFC can reduce the shortcut flow rate, prevent particle entrapment in the vortex finder, and thus enhance the collection efficiency.

\section{Effect of the Cone Angle}

Studies have shown that the cone dimension and cone angle have critical effects on cyclone performance characteristics in a common cyclone (Xiang et al., 2001; Brar et al., 2015b). The effects of the cone angle on the separation performance were investigated in the CFCs at the range of the cone angle from $18^{\circ}$ to $30^{\circ}$. Figs. 6(a) and 6(b) show the variations with respect to cone angle of calculated separation efficiencies and pressure drops under different inlet velocities, respectively. The figures show that the separation efficiency first increases, and then decreases with the increase of the cone angle within the range of the inlet velocity from 22 to $30 \mathrm{~m} \mathrm{~s}^{-1}$, while the pressure drop shows a decline first followed by a rise. The turning point for both is around $24^{\circ}$ for the cone angle. Such results differ from the results in a common cyclone, in which increasing cone angle leads to increasing the static pressure and the total pressure drop (Hamdy et al., 2017). The main cause should be that cone zone has an important contribution for the pressure drop in a common cyclone, while it has a lesser effect in the CFC, due to the path difference of the main gas flow in the two kinds of cyclones.

\section{Particle Trajectory}

Fig. 7 shows the trajectories of particles with different diameters. As shown, the particles with diameters of $1-6 \mu \mathrm{m}$
)

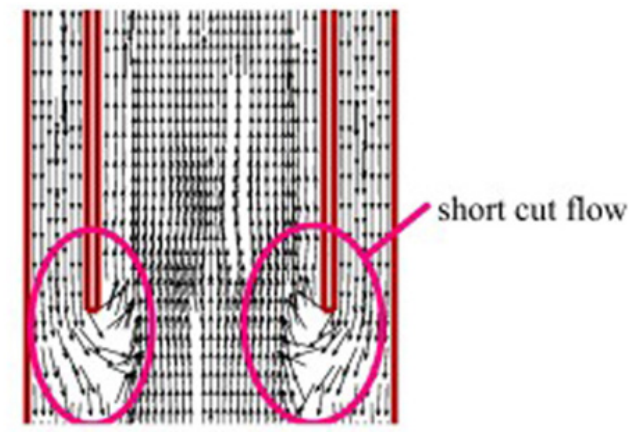

Fig. 5. The velocity vector near the vortex finder entrance. (a) CFC and (b) a common cyclone (Xu et al., 2016). 


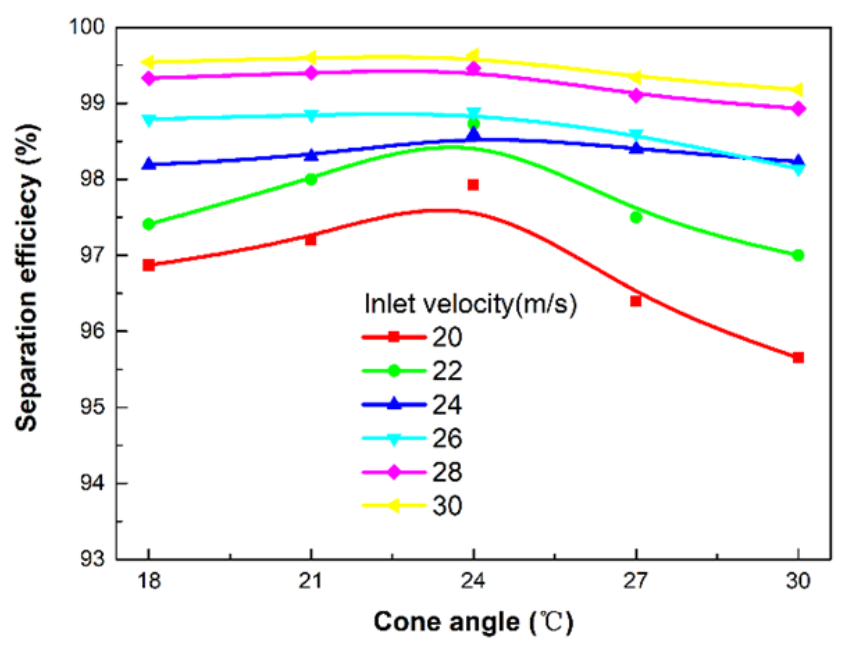

a)



b)

Fig. 6. The effect of cone angle on the separation performance. (a) Separation efficiency and (b) pressure drop.
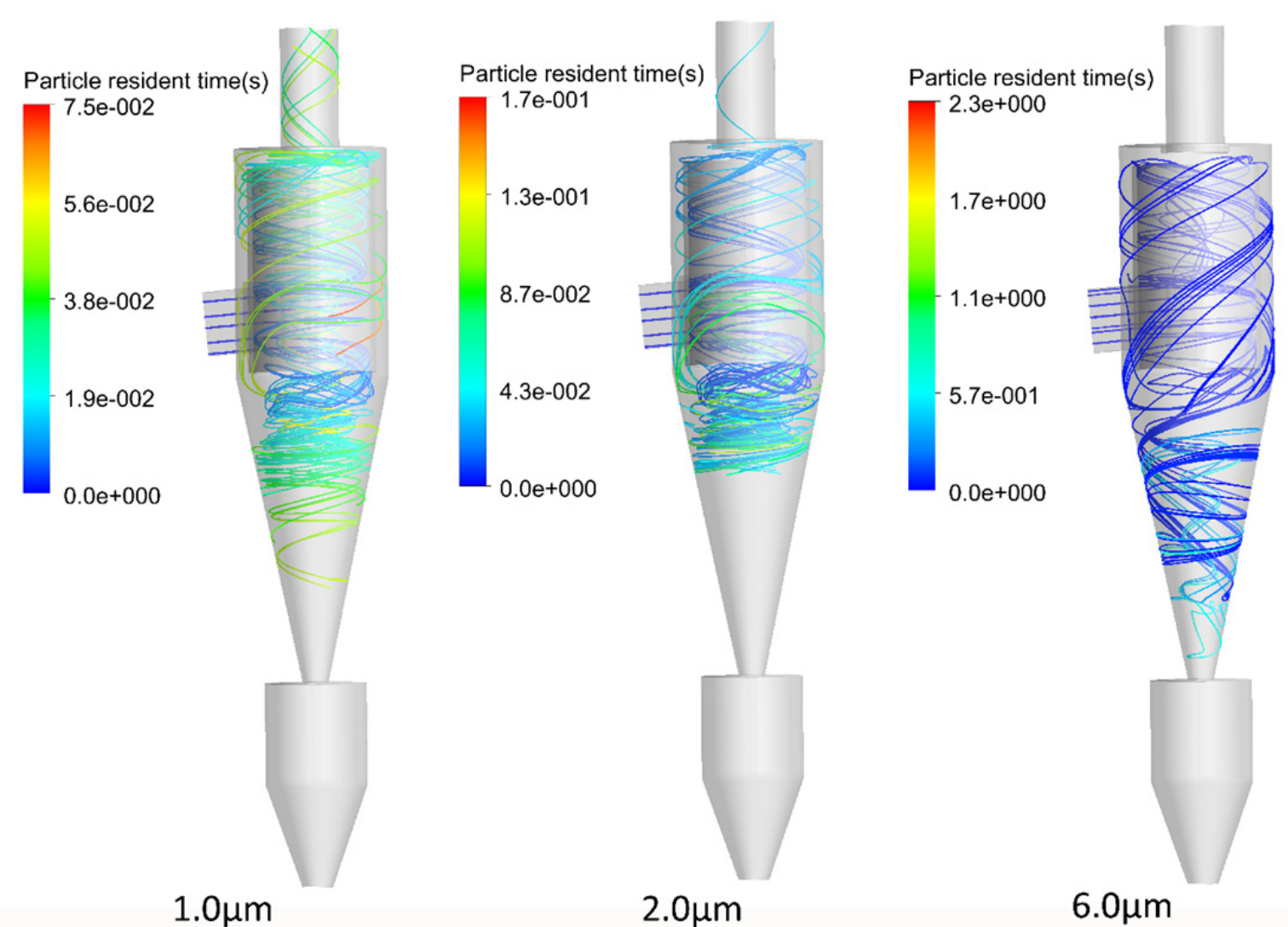

Fig. 7. Particle with different diameter trajectories (particle diameters from left to right are $1.0 \mu \mathrm{m}, 2.0 \mu \mathrm{m}, 6.0 \mu \mathrm{m}$ ).

all flow into the inner cylinder and helically move upward around the inner cylinder wall. They move into the annular space by centrifugal force from the top of the inner cylinder and continue to flow downward to the cone zone. Afterwards, different diameter particles have different paths.

Many 1- $\mu \mathrm{m}$-diameter particles are carried into the centre of the inner cylinder and soon flow out of the cyclone from the vortex finder. The larger particles, with $6 \mu \mathrm{m}$ diameter, flow further down to the cone zone and ash collector, then can be trapped. Most 2- $\mu$ m-diameter particles spin down to the conical part and then keep spinning near the wall in a certain plane. This is probably because, with the decease of the radius at the conical zone, the tangential and axial gas velocities also increase. However, the increase rate of the tangential velocity is less than that of the axial component due to the former being almost inversely proportional to the radius, while the latter is roughly inversely proportional to the square root of the radius. Thus, the centrifugal force 
and the axial component force on the particle all increase, but the former increases less. The particle will move up when the axial component force is larger than the sum of the gravity and the axial component of the gas drag force. If the axial component force is equal to the sum of the gravity and the axial component of the gas drag force, the particle will keep spinning at a certain height (Wang et al., 2006). However, a small amount of $1-\mu \mathrm{m}$-diameter particles also keep spinning at a certain height. And very few $2-\mu \mathrm{m}-$ diameter particles would escape from the outlet.

\section{CONCLUSION}

The Reynolds stress model was used to simulate the flow pattern and flow velocity distribution in a CFC, which has an inner cylinder situated inside the main cylinder. The model's applicability was verified by good agreement between the calculated and measured tangential velocities, pressure drops, and separation efficiencies. The experimental results (Wang et al., 2010) confirmed that the CFC exhibited a higher separation efficiency and a lower pressure drop than a conventional cyclone. Numerical results were used to explain why the CFC has superior performance.

First, the flow pattern shows that the major portion of the gas flows directly out of the CFC through the vortex finder, which causes a smaller pressure drop. Second, the $\mathrm{CFC}$ reduces the shortcut flow rate near the vortex finder entrance. About $10 \%$ of the total flow escapes the cyclone through the vortex finder after being separated by the inertial force around the top of the inner cylinder, when the diametric ratio of the inner cylinder to the main cylinder is 0.65 . The diametric ratio affects the separation performance, an issue that will be studied in subsequent work. The numerical results also indicate that a cone angle of $24^{\circ}$ produces optimal separation performance.

\section{ACKNOWLEDGEMENTS}

This work was sponsored by National Natural Science Foundation of China (No. 21276132) and a Project of Shandong Province Higher Educational Science and Technology Program (No. J17KA107).

\section{NOMENCLATURE}

$\begin{array}{ll}a & \text { Cyclone inlet width }(\mathrm{m}) \\ b & \text { Cyclone inlet height }(\mathrm{m}) \\ D & \text { Cyclone body diameter }(\mathrm{m}) \\ D \mathrm{c} & \text { Cyclone dust outlet diameter }(\mathrm{m}) \\ D \mathrm{i} & \text { Cyclone inner cylinder diameter }(\mathrm{m}) \\ D \mathrm{o} & \text { Cyclone gas outlet diameter }(\mathrm{m}) \\ H & \text { Cyclone cylinder height }(\mathrm{m}) \\ H \mathrm{c} & \text { Cyclone cone height }(\mathrm{m}) \\ v & \text { Gas inlet velocity }\left(\mathrm{m} \mathrm{s}^{-1}\right) \\ D \mathrm{p} & \text { Particle diameter } \\ \mathrm{C}_{\mathrm{D}} & \text { Drag coefficient } \\ \mathrm{g} & \text { Gravitational acceleration } \\ \rho_{\mathrm{p}} & \text { Particle density }\end{array}$

$\begin{array}{ll}\text { Re } & \text { Relative Reynolds number } \\ u & \text { Gas velocity } \\ u_{p} & \text { Particle velocity }\end{array}$

\section{REFERENCES}

Abrahamson, J., Jones, R., Lau, A. and Reveley, S. (2002). Influence of entry duct bends on the performance of return-flow cyclone dust collectors. Powder Technol. 123: $126-137$.

Avci, A. and Karagoz, I. (2003). Effects of flow and geometrical parameters on the collection efficiency in cyclone separators. J. Aerosol Sci. 34: 937-955.

Bernardo, S., Mori, M., Peres, A.P. and Dionísio, R.P. (2006). 3-D computational fluid dynamics for gas and gas-particle flows in a cyclone with different inlet section angles. Powder Technol. 162: 190-200.

Bhaskar, K.U., Murthy, Y.R., Raju, M.R., Tiwari, S., Srivastava, J.K. and Ramakrishnan, N. (2007). CFD simulation and experimental validation studies on hydrocyclone. Miner. Eng. 20: 60-71.

Boysan, F., Ayers, W.H. and Swithenbank, J. (1982). A fundamental mathematical modelling approach to cyclone design. Chem. Eng. Res. Des. 60: 222-230.

Brar, L.S., Sharma, R.P. and Dwivedi, R. (2015a). Effect of vortex finder diameter on flow field and collection efficiency of cyclone separators. Part. Sci. Technol. 33: 34-40.

Brar, L.S., Sharma, R.P. and Elsayed, K. (2015b). The effect of the cyclone length on the performance of stairmand high-efficiency cyclone. Powder Technol. 286: 668-677.

Chmielniak, T. and Bryczkowski, A. (2000). Method of calculation of new cyclone-type separator with swirling baffle and bottom take off of clean gas - Part I: theoretical approach. Chem. Eng. Process. 39: 441-448.

Chmielniak, T. and Bryczkowski, A. (2001). Method of calculation of new cyclone-type separator with swirling baffle and bottom take off of clean gas - Part II: Experimental verification. Chem. Eng. Process. 40: 245-254.

Chuah, T.G., Gimbun, J. and Choong, T.S.Y. (2006). A CFD study of the effect of cone dimensions on sampling aerocyclones performance and hydrodynamics. Powder Technol. 162: 126-132.

Derksen, J.J. and Van den Akker, H.E.A. (2000). Simulation of vortex core precession in a reverse-flow cyclone. AIChE J. 46: 1317-1331.

Derksen, J.J. (2003). Separation performance predictions of a stairmand high-efficiency cyclone. AIChE J. 49: 1359-1371.

El-Batsh, H.M. (2013). Improving cyclone performance by proper selection of the exit pipe. Appl. Math. Modell. 37: 5286-5303.

Elsayed, K. and Lacor, C. (2010). Optimization of the cyclone separator geometry for minimum pressure drop using mathematical models and CFD simulations. Chem. Eng. Sci. 65: 6048-6058.

Elsayed, K. and Lacor, C. (2013). The effect of cyclone 
vortex finder dimensions on the flow pattern and performance using LES. Comput. Fluids 71: 224-239.

Fluent Inc. (2005). Fluent 6.2 user's guide. Fluent Inc., Lebanon, NH, USA.

Gimbun, J., Chuah, T.G., Fakhru'l-Razi, A. and Choong, T.S.Y. (2005). The influence of temperature and inlet velocity on cyclone pressure drop: A CFD study. Chem. Eng. Process. 44: 7-12.

Griffiths, W.D. and Boysan, F. (1996). Computational fluid dynamics (CFD) and empirical modelling of the performance of a number of cyclone samplers. $J$. Aerosol Sci. 27: 281-304.

Gupta, A. and Kumar, R. (2007). Three-dimensional turbulent swirling flow in a cylinder: Experiments and computations. Int. J. Heat Fluid Flow 28: 249-261.

Hamdy, O., Bassily, M.A., El-Batsh, H.M. and Mekhail, T.A. (2017). Numerical study of the effect of changing the cyclone cone length on the gas flow field. Appl. Math. Modell. 46: 81-97.

Hoekstra, A.J., Derksen, J.J. and Van Den Akker, H.E.A. (1999). An experimental and numerical study of turbulent swirling flow in gas cyclones. Chem. Eng. Sci. 54: 2055-2065.

Hsu, C.W., Huang, S.H., Lin, C.W., Hsiao, T.C., Lin, W.Y. and Chen, C.C. (2014). An experimental study on performance improvement of the stairmand cyclone design. Aerosol Air Qual. Res. 14: 1003-1016.

Jiao, J., Zheng, Y., Wang, J. and Sun, G. (2008). Experimental and numerical investigations of a dynamic cyclone with a rotary impeller. Chem. Eng. Process. Process Intensif. 47: 1861-1866.

Jo, Y., Tien, C. and Ray, M.B. (2000). Development of a post cyclone to improve the efficiency of reverse flow cyclones. Powder Technol. 113: 97-108.

Kim, J. and Lee, K. (1990). Experimental study of particle collection by small cyclones. Aerosol Sci. Technol. 12: 1003-1015.

Lapple, C. (1951). Processes use many collector types. Chem. Eng. 58: 144-151.

Lee, J.W., Yang, H.J. and Lee, D.Y. (2006). Effect of the cylinder shape of a long-coned cyclone on the stable flow-field establishment. Powder Technol. 165: 30-38.

Lim, K.S., Kwon, S.B. and Lee, K.W. (2003). Characteristics of the collection efficiency for a double inlet cyclone with clean air. J. Aerosol Sci. 34: 10851095.

Lim, K.S., Kim, H.S. and Lee, K.W. (2004a). Characteristics of the collection efficiency for a cyclone with different vortex finder shapes. J. Aerosol Sci. 35: 743-754.

Lim, K.S., Kim, H.S. and Lee, K.W. (2004b). Comparative performances of conventional cyclones and a double cyclone with and without an electric field. J. Aerosol Sci. 35: 103-116.

Lin, C.W., Chen, T.J., Huang, S.H., Kuo, Y.M., Gui, H.Q. and Chen, C.C. (2018). Effect of aerosol loading on separation performance of $\mathrm{PM}_{2.5}$ cyclone separators. Aerosol Air Qual. Res. 18: 1366-1374.

Liu, D., Wang, P., Hsiao, T.C. and Chen, D.R. (2018).
Small cyclones with conical contraction bodies. Aerosol Air Qual. Res. 18: 2519-2528.

Ma, L., Fu, P., Wu, J., Wang, F., Li, J., Shen, Q. and Wang, H. (2015). CFD simulation study on particle arrangements at the entrance to a swirling flow field for improving the separation efficiency of cyclones. Aerosol Air Qual. Res. 15: 2456-2465.

Martignoni, W.P., Bernard, S. and Quintani, C.L. (2007). Evaluation of cyclone geometry and its influence on performance parameters by computational fluid dynamics (CFD). Braz. J. Chem. Eng. 24: 83-94.

Misiulia, D., Andersson, A.G. and Lundstrom, T.S. (2017). Effects of the inlet angle on the collection efficiency of a cyclone with helical-roof inlet. Powder Technol. 305: 48-55.

Obermair, S., Woisetschlager, J. and Staudinger, G. (2003). Investigation of the flow pattern in different dust outlet geometries of a gas cyclone by laser doppler anemometry. Powder Technol. 138: 239-251.

Ogawa, A., Hironaka, A., Kato, T. and Seito, O. (1991). Velocity distributions of gas flow and the separation efficiency of the semi-spherical cyclone. Adv. Powder Technol. 2: 191-212.

Plomp, A., Beumer, M.I.L. and Hoffmann, A. (1996). PostCyclone (PoC) an approach to a better efficiency of dustcyclones. J. Aerosol Sci. 27: S631-S632.

Qian, F. and Zhang, M. (2005). Study of the natural vortex length of a cyclone with response surface methodology. Comput. Chem. Eng. 29: 2155-2162.

Raoufi, A., Shams, M., Farzaneh, M. and Ebrahimi, R. (2008a). Numerical simulation and optimization of fluid flow in cyclone vortex finder. Chem. Eng. Process. Process Intensif. 47: 128-137.

Raoufi, A., Shams, M., Farzaneh, M. and Ebrahimi, R. (2008b). Numerical simulation and optimization of fluid flow in cyclone vortex finder. Chem. Eng. Process. Process Intensif. 47: 128-137.

Raoufi, A., Shams, M. and Kanani, H. (2009). CFD analysis of flow field in square cyclones. Powder Technol. 191: 349-357.

Siadaty, M., Kheradmand, S. and Ghadiri, F. (2017). Study of inlet temperature effect on single and double inlets cyclone performance. Adv. Powder Technol. 28: 14591473.

Slack, M.D., Prasad, R.O., Bakker, A. and Boysan, F. (2000). Advances in cyclone modelling using unstructured grids. Chem. Eng. Res. Des. 78: 1098-1104.

Stairmand, C. (1951). The design and performance of cyclone separators. Chem. Eng. Res. Des. 29: 356-383.

Tian, Y.H., Ji, Z.L. and Shi, M.X. (1992). The flow field of PV cyclone separator. J. Univ. Pet. China 16: 54-60.

Wang, B., Xu, D.L., Chu, K.W. and Yu, A.B. (2006). Numerical study of gas-solid flow in a cyclone separator. Appl. Math. Modell. 30: 1326-1342.

Wang, W., Zhang, P., Wang, L., Chen, G., Li, J. and Li, X. (2010). Structure and performance of the circumfluent cyclone. Powder Technol. 200: 158-163.

Xiang, R., Park, S.H. and Lee, K.W. (2001). Effects of cone dimension on cyclone performance. J. Aerosol Sci. 
32: 549-561.

Xiang, R.B. and Lee, K.W. (2005). Numerical study of flow field in cyclones of different height. Chem. Eng. Process. 44: 877-883.

Xu, W.W., Li, Q., Wang, J.J. and Jin, Y.H. (2016). Performance evaluation of a new cyclone separator Part II simulation results. Sep. Purif. Technol. 160: 112116.

Yang, J.X., Sun, G.G. and Gao, C.Z. (2013). Effect of the inlet dimensions on the maximum-efficiency cyclone height. Sep. Purif. Technol. 105: 15-23.

Yoshida, H., Fukui, K., Yoshida, K. and Shinoda, E. (2001). Particle separation by linoya's type gas cyclone. Powder Technol. 118: 16-23.

Yoshida, H., Hayase, Y., Fukui, K. and Yamamoto, T. (2012). Effect of conical length on separation performance of sub-micron particles by electrical hydro- cyclone. Powder Technol. 219: 29-36.

Zhao, B., Shen, H. and Kang, Y. (2004). Development of a symmetrical spiral inlet to improve cyclone separator performance. Powder Technol. 145: 47-50.

Zhao, B., Su, Y. and Zhang, J. (2006). Simulation of gas flow pattern and separation efficiency in cyclone with conventional single and spiral double inlet configuration. Chem. Eng. Res. Des. 84: 1158-1165.

Zhou, L.X. and Soo, S.L. (1990). Gas-solid flow and collection of solids in a cyclone separator. Powder Technol. 63: 45-53.

Received for review, June 14, 2018 Revised, December 5, 2018 Accepted, January 11, 2019 